\title{
Lidil
}

Revue de linguistique et de didactique des langues

$50 \mid 2014$

Variation stylistique et diversité des contextes de socialisation

\section{L'éveil à la variation phonétique en didactique du français langue étrangère : enjeux et outils}

Raising the Awareness of Phonetic Variation in the Teaching of French as a Foreign Language: Issues and Tools

\section{Roberto Paternostro}

\section{OpenEdition}

\section{Journals}

Édition électronique

URL : http://journals.openedition.org/lidil/3587

DOI : $10.4000 /$ lidil.3587

ISSN : 1960-6052

\section{Éditeur}

UGA Éditions/Université Grenoble Alpes

\section{Édition imprimée}

Date de publication : 15 décembre 2014

Pagination : 105-124

ISBN : 978-2-84310-287-5

ISSN : $1146-6480$

\section{Référence électronique}

Roberto Paternostro, «L'éveil à la variation phonétique en didactique du français langue étrangère : enjeux et outils », Lidil [En ligne], 50 | 2014, mis en ligne le 15 juin 2016, consulté le 30 avril 2019. URL: http://journals.openedition.org/lidil/3587 ; DOI : 10.4000/lidil.3587 


\title{
L'éveil à la variation phonétique en didactique du français langue étrangère : enjeux et outils
}

\author{
Roberto Paternostro*
}

\begin{abstract}
RÉSUMÉ
Cet article se donne pour objectif de réfléchir aux raisons pour lesquelles la phonétique est le parent pauvre de la didactique du FLE. Il propose d'intégrer des indices phoniques de la variation stylistique à l'enseignement du français. À partir d'une expérience issue d'un terrain didactique, l'auteur se penche sur le «transcodage» en tant qu'outil d'éveil à la variation phonétique en FLE.
\end{abstract}

\section{ABSTRACT}

This paper attempts to understand why pronunciation is the poor relative of the teaching of French as a foreign language. It examines how phonetic indices of stylistic variation could be integrated in the French language teaching. On the basis of a field study, the author discusses "transcoding" as a tool for awakening phonetic variation in French as a foreign language.

\section{Introduction}

Les manuels d'enseignement de la prononciation en français langue étrangère (FLE) ainsi que le matériel utilisé en laboratoire de langue proposent de plus en plus un travail sur l'expressivité et plus rarement des activités portant sur les traits phonétiques du français ordinaire, comme les phénomènes d'assimilation ou de simplification : je sais pas [Sepa], peut-être [ptet]. Néanmoins, ils se limitent souvent à la description d'aspects prosodiques et articulatoires de base, à partir d'extraits sonores joués par des acteurs, peu spontanés, qui constituent le seul contexte offert aux apprenants.

* ELCF - Université de Genève. 
Force est de constater l'absence de la variation phonétique, notamment pour le style. Cet article se donne pour objectif de réfléchir aux raisons de cette absence et à l'intérêt d'un éveil à la variation phonétique en FLE.

\section{La variation phonétique en FLE : les raisons d'une absence}

En tant que locuteur «étranger ${ }^{1} »$ du français, je me souviens du moment où des amis parisiens m'ont fait remarquer que mon français était «trop parfait» pour faire français. Ils pointaient certains indices phoniques : l'absence d'assimilation ou la réalisation de la liaison facul-

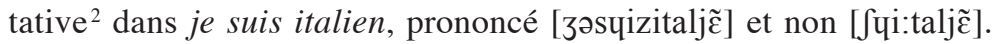
Bien qu'ayant entendu de telles réalisations, je ne les avais pas croisées dans un apprentissage spécifique, et n'osais pas alors m'aventurer dans le domaine inaccessible du non standard, à mon sens réservé aux «natifs ${ }^{3} »$. L'emploi constant du standard me valait d'ailleurs des remarques que je considérais comme flatteuses : «tu parles comme un prof », «tu parles très bien, on dirait un livre».

Les cours de FLE privilégient en effet l'apprentissage du standard comme le français par défaut ${ }^{4}$, reléguant la langue ordinaire 5 à l'ap-

1. Pour l'auteur de cet article, qui est de langue première italienne, le français est une langue «étrangère». La catégorisation d'étrangère est pourtant problématique dès que l'apprenant commence à se familiariser avec une langue, qui n'est plus alors «étrangère» (Gadet, 2012).

2. Gadet (1997, p. 71) souligne le caractère d'«indicateur sociolinguistique très fort» de la liaison, sans doute en lien avec le rapport oral/écrit, l'écrit étant associé au prestige.

3. Berruto (2003) a montré la vacuité des notions de «locuteur natif» et «langue maternelle», impossibles à définir en termes positifs. Quant aux notions de L1 et de L2, langue «première» et «seconde», qui seraient à préférer à «langue maternelle» et «étrangère», elles ne sont pas moins problématiques.

4. Nous faisons allusion à l'idéologie du standard, qui considère la forme normée comme la langue de référence, pratiquée par tous les locuteurs appartenant aux couches sociales favorisées et représentée principalement par l'écrit (Gadet, 2007, p. 17-18).

5. Le français ordinaire n'est pas le français parlé ni le français populaire. «C'est davantage le français familier, celui dont chacun est porteur dans son fonctionnement quotidien, dans le minimum de surveillance sociale : la langue de tous les jours.» (Gadet, 1997, p. 3) 
prentissage sur le tas, si les apprenants ont l'occasion de côtoyer des natifs (voir Regan et coll., 2009) ${ }^{6}$, ce qui a pour effet de produire des locuteurs qui donnent l'impression de parler mieux que les natifs. Par ailleurs, la problématique de la place et du statut que le standard occupe dans l'enseignement concerne aussi le français langue maternelle (FLM). Guerin (2010) a ainsi attiré l'attention sur ce qu'elle appelle l'«outrelangue» des enseignants à l'école, à qui l'institution demande d'actualiser «une langue au-delà de la langue», ne s'inscrivant dans rien d'observable.

La norme $^{7}$ est le premier input pour des apprenants de FLE, et reste souvent la seule actualisation de la langue à laquelle ils ont accès (voir, entre autres, Valdman, 1989; Detey \& Racine, 2012) ${ }^{8}$. Contrairement aux locuteurs L1, en effet, pour qui le contact avec la norme se fait à l'école et qui sont d'abord confrontés à la langue ordinaire de leur entourage, l'apprenant, au début, n'a généralement de contact direct qu'avec la langue de l'enseignant en cours. Il risque alors de croire qu'il s'agit là de «la» langue française. Bien qu'il ait un écho de ce qui se parle hors de la classe, notamment par les documents authentiques, l'internet, les médias, etc., le français ordinaire demeure souvent marginal, un luxe.

Malgré des efforts, ces dernières années, pour intégrer la variation à l'enseignement du français, notamment par l'introduction d'accents francophones (Detey et coll., 2010) et par l'intérêt pour le lexique familier et l'intonation expressive (Callamand, 1973; Léon, 1993), la variation phonétique demeure le parent pauvre des cours de FLE.

\subsection{Les indices phoniques du style ou la variation invisible}

L'apprenant ne dispose pas de toutes les références sociales nécessaires pour décrypter les nuances de la variation stylistique. C'est donc par l'apprentissage qu'il doit se les construire (Gadet, 2001). Bien que le

6. Bien que l'intérêt d'un apprentissage en immersion ne soit pas remis en cause, tous les apprenants n'ont pas l'occasion de faire un séjour linguistique à l'étranger.

7. La notion de norme évoquée ici recoupe la définition de standard donnée dans la note 4 .

8. La «primauté» de la norme nous parait tout à fait légitime, vu le caractère particulier de l'apprentissage d'une langue étrangère. Cependant, cela ne justifie pas le rôle marginal que l'on réserve à l'apprentissage du non-standard. 
phonique constitue un indicateur saillant pour le diaphasique (Gadet, 2007, p. 10) ${ }^{9}$, il fait peu l'objet d'un apprentissage explicite en cours ou dans les manuels. La variation stylistique est le plus souvent abordée à partir d'exemples lexicaux, domaine dans lequel le changement de registre semble plus évident, car chaque item semble renvoyer à un style : argent/fric, femme/meuf, policier/flic, etc. (entre autres, Charliac \& Motron, 2006, p. 109).

La variation phonétique est complexe à définir et à enseigner, et difficile à assigner à un registre. Les assimilations et les simplifications de la chaine parlée ou les liaisons facultatives, souvent associées au registre familier, apparaissent de fait autant dans une interaction entre amis que dans une conférence ou dans la parole publique (voir Lodge et coll., 1997). Les traits phonétiques ne peuvent donc pas être immédiatement associés à un style de parole ou à une hiérarchie sociale. Ils sont plutôt à situer sur un continuum, et fonctionnent par cumul selon des paramètres complexes, en fonction de la distance/proximité communicationnelle entre locuteurs (Koch \& Esterreicher, 2001) ${ }^{10}$. C'est leur combinaison et leur fréquence dans une situation - et non un trait isolé - qui créent un accent ou un style.

Ainsi, un contour intonatif montant-descendant, souvent associé à un accent de banlieue, passe inaperçu lorsqu'il est produit en contexte «inhabituel» et ne s'accompagne pas d'autres indices regardés comme typiques de jeunes de banlieue (Gadet \& Paternostro, 2013). Dès lors, les traits phonétiques peuvent être appréhendés en tant qu'ensemble non hiérarchisé et non précatégorisé, ressource hétérogène pour la langue en contexte.

Dans une telle architecture, le standard n'est donc plus la référence, mais une actualisation de la langue associée à la distance communi-

9. Le diaphasique, appelé aussi variation stylistique ou situationnelle, concerne la différenciation linguistique selon l'usage. Voir Gadet (2007, p. 10), pour une définition.

10. Pour ces auteurs, l'oral et l'écrit, abusivement associés à l'informel et au formel, ne sont qu'un médium. La distinction formel/informel relève quant à elle du conceptionnel, actualisé dans un continuum en fonction de paramètres définis par les pôles de la proximité et de la distance communicative. Les formes de l'oral (dont le phonique), souvent considérées comme informelles voire «fautives», sont donc à situer sur le même continuum, participant ainsi à l'actualisation de la langue dans la proximité et dans la distance, au même titre que les formes de l'écrit. 
cative, qui se caractérise par la sélection d'unités linguistiques, dont l'interprétation est indépendante d'un savoir partagé (Guerin, 2008). Ce n'est plus «le» français, mais l'une de ses actualisations. Le but de l'apprentissage n'est donc plus la transmission de la norme, mais la maitrise et le maniement, en perception (et parfois, en production), de toutes les potentialités du français ${ }^{11}$.

\section{L'intérêt du corpus Multicultural Paris French (MPF) ${ }^{12}$ pour la didactique du FLE}

Nul ne doute aujourd'hui du rôle des corpus oraux dans l'enseignement du FLE (Boulton \& Tyne, 2014). Pourtant, il y a un décalage entre ce que montrent les corpus et la façon dont les faits de langues sont présentés dans les manuels et les cours de FLE (Debaisieux \& Boulton, 2007; Detey, 2010). Il parait dès lors légitime de se demander ce que serait l'intérêt d'un corpus comme MPF pour la didactique du FLE. Nous avons montré (Paternostro, 2014) que ce qu'on appelle «accent de banlieue» est moins la marque d'une marginalité linguistique et sociale que l'usage de la langue dans un cadre de proximité communicationnelle. Les interactions qui se tissent en banlieue semblent favoriser l'émergence d'un «style de parole emphatique» (voir Selting, 1994), caractérisé par des traits de prononciation exprimant la connivence et l'implication des locuteurs. «La vie dans la cité, c'est chaleureux!», dit un informateur.

Parmi les traits phoniques concernés, la plupart coïncident avec ce que Gadet (1997, p. 95-106) appelle facilités de prononciation, caractéristiques du français ordinaire : assimilations de sonorité (je sais pas [Sepa]); assimilations du mode d'articulation (maintenant [mẽnã]); harmonie vocalique (surtout [sustu]); simplification de groupes consonantiques complexes, à l'intérieur du mot (quelque chose [kekfoz], il dit [idi]), ou en finale (quatre [kat]); réductions vocaliques (tu as [ta], c'était [ste]). À cette liste, non exhaustive, on peut ajouter : chute du e muet, non-réalisation des liaisons facultatives, liaisons inhabi-

11. Ce que le CECR (Conseil de l'Europe, 2005) n'a pas manqué de souligner, en introduisant une approche plus fonctionnelle, liée aux actes de langage, et la séparation des compétences.

12. Le corpus MPF est issu du projet Multicultural Paris French (ANR-09FRBR-037-01), coordonné par Gadet (voir Gadet \& Guerin, 2012). 
tuelles, contours intonatifs emphatiques, affrication et palatalisation des consonnes occlusives dentales et vélaires devant /i/ et /y/ (étude [êtfyd],

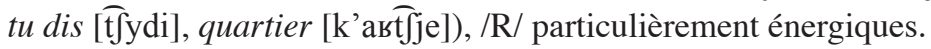

D'autres éléments, syntaxiques, discursifs et lexicaux, peuvent accompagner ces traits de prononciation. Par exemple, les phatiques et les ponctuants (ouais, quoi, bon, ben), l'omission du ne de négation, les hésitations (euh), les amorces ( $t a-t a-t a b l e)$, les reformulations, etc. Ces phénomènes, typiques de l'oral, peuvent aussi fonctionner en marques de connivence en communication ordinaire. Même s'ils peuvent paraitre marginaux pour notre objectif actuel, ils sont au contraire au cœur de l'interaction : ils favorisent ou reflètent la proximité communicationnelle, la co-construction de l'interaction, et ré-assurent la collaboration des interlocuteurs (André \& Tyne, 2010).

Les interactions dans le corpus MPF constituent un observatoire privilégié sur un certain aspect de la langue ordinaire (des interactions entre jeunes), et permettent aux apprenants de se confronter à des traits linguistiques illustrant la proximité et la connivence, insolites pour un cours de FLE, dans le but d'un éveil à la variation.

\section{Le transcodage, outil d'éveil à la variation phonétique}

Dans un cadre de pédagogie de l'écoute (Lhote, 1995; Guimbretière, 1992 $)^{13}$ et de data-driven learning (Johns, 1993), nous proposons une activité didactique visant à sensibiliser les apprenants aux indices phoniques de la variation stylistique ${ }^{14}$. À partir d'extraits d'interactions authentiques ${ }^{15} \mathrm{du}$ corpus MPF, les apprenants pourront se familiariser avec un style de parole illustrant la proximité. Après une phase d'écoute et d'observation d'un extrait sonore, les apprenants sont appelés à transcrire ce qu'ils ont cru percevoir. Nous appelons le passage par la trans-

13. Ce terme est ici préféré à «correction phonétique», à la fois pour éviter «correction» qui suppose la faute, et pour souligner l'importance de l'écoute dans l'apprentissage de la prononciation.

14. Nous soulignons que la production n'est pas envisagée ici. Seule la compréhension est visée.

15. Par «authentiques», nous entendons des interactions qui ne sont ni jouées par des acteurs ni produites suivant un script ou un but précis. Il s'agit d'extraits d'entretiens qui respectent l'écologie de l'évènement en cours et qui sont jugés naturels à la fois par les interactants et par le chercheur. 
cription «transcodage ${ }^{16}$ », à la fois pour écarter l'idée de scripturalité et pour marquer qu'il s'agit d'une transposition de l'oral vers l'écrit. Il se fait selon l'orthographe standard, pour assurer la lisibilité du texte, sauf pour la ponctuation, marquée par des signes spécifiques à l'oral ${ }^{17}$. Le passage par l'écrit n'est qu'une étape passagère, mais nécessaire, qui permet à l'apprenant de focaliser son attention sur le phonique tout en renforçant sa capacité d'écoute et d'observation de la langue en contexte $^{18}$.

L'idée nous est venue lors de la transcription du corpus MPF. Le fait de consacrer du temps à la transcription de données orales relevant de la proximité communicationnelle a affiné notre perception de la variation (voir aussi Tyne, 2009a). La transcription a peu à peu changé notre écoute et nous a aidé à percevoir des détails de la chaine parlée, qui apparait souvent comme un flux ininterrompu aux oreilles des apprenants. Elle nous a aussi donné une clé pour appréhender le paysage sonore (Lhote, 1990), mais aussi géo-social et culturel, de la région parisienne.

\subsection{Le transcodage en FLE : avantages et inconvénients}

Le transcodage en tant qu'activité didactique en FLE joue un rôle initiatique, d'observation et de familiarisation avec la langue ordinaire. Pour le phonique, il constitue un outil d'éveil à la variation, favorisant l'écoute et le repérage de traits de prononciations dans un contexte de proximité et de connivence. La production n'est donc pas visée ici (voir Valdman, 2000).

À partir d'un travail global sur l'oralité, visant au développement de stratégies d'analyses et de segmentation de la chaine parlée, l'apprenant est amené à se focaliser sur la dimension segmentale et supra-

16. Malgré la pertinence du terme transcodage pour une réflexion didactique, nous sommes conscient de son opacité sémantique pour les apprenants. C'est pourquoi nous l'utilisons pour l'activité globale et gardons «transcription» quand l'acte de transcrire est en cause.

17. (.) et (..) marquent respectivement une pause brève et longue; le point indique une frontière majeure d'énoncé; le tiret marque les amorces de mots ou les répétitions.

18. Vu le rôle de «charnière» joué par l'écrit et le choix d'une transcription en orthographe standard, nous n'abordons pas ici l'impact d'une telle activité sur l'apprentissage de l'écriture. 
segmentale, favorisant ainsi l'enrichissement de son système phonéticophonologique.

La transcription, sous différentes formes, n'est pas une activité inconnue en didactique des langues (Lebre-Peytard et coll., 1981; Biggs \& Dalwood, 1976; Lynch, 2007; Stillwell et coll., 2010; Pimsleur, 1979; Boulton \& Tyne, 2014, pour une synthèse). Tyne (2009a) rapporte une expérience impliquant des étudiants britanniques dans la collecte, la transcription et l'analyse de leur propre corpus de français parlé. Le contact avec les données, et leur transcription, s'est avéré une réelle occasion d'apprentissage, surtout pour ce qui est de la prise de conscience de la variation.

La transcription n'est néanmoins pas proposée par la plupart des manuels de prononciation, qui offrent surtout un travail de phonie/graphie. Dans le transcodage, au contraire, la graphie ne constitue pas un but en soi, mais un passage dans un aller-retour, de l'oral à l'oral par l'écrit. Pour reprendre la terminologie de Lhote (1990), le transcodage intervient entre les étapes d'ancrage et de repérage, et vise à renforcer la compréhension. L'attention portée au détail grâce au passage par l'écrit, associée à la possibilité d'écouter à loisir, permettent en effet de gérer la diversité en perception. Au fur et à mesure que le brouhaha initial s'éclaircit, l'apprenant découvre le multiple et la variation dans ce qu'il croyait être unique et invariable.

L'observation en contexte introduite par le transcodage d'interactions brèves et authentiques permet alors de contourner la difficulté à dresser une liste de traits de prononciation qui corresponde à un style de parole prédéterminé, de façon à ce que l'apprenant se rende compte des traits reflétant une langue de tous les jours.

Le transcodage rappelle la dictée à cause du passage de l'oral à l'écrit, mais il en diffère de deux façons essentielles. L'extrait à transcrire n'est pas un écrit oralisé, suivant des réalisations phonétiques artificielles du texte lu, comme c'est le cas dans la dictée, mais un échantillon d'interactions authentiques. Contrairement à la dictée, permettant de tester la correction de l'écrit, le transcodage est une démarche descriptive, qui ne fera pas l'objet d'une évaluation de la part de l'enseignant $^{19}$. L'apprenant pourra néanmoins se confronter au corrigé fourni

19. Le transcodage, en fait, renoue avec ce qu'était la dictée pour les phonéticiens pédagogues au XIX ${ }^{\mathrm{e}}$ siècle, à savoir une activité d'écoute (voir Galazzi, 2002). 
par l'enseignant, permettant de mesurer sa capacité à comprendre. Il s'agit d'une auto-évaluation qui nait de la comparaison, d'une découverte. Déchargé de son orientation prescriptive, l'écrit permet alors à l'apprenant de retrouver l'unité qu'il avait cru perdue dans la multiplicité du variable : c'est un véritable appui pour la mise en place d'une passerelle entre l'homogénéité du signe graphique et le foisonnement des réalisations orales.

Le passage par l'orthographe standard constitue aussi une sensibilisation aux différences entre oral et écrit permettant une rupture par rapport à la dichotomie oral/écrit : même des formes orales souvent considérées comme fautives peuvent s'écrire (voir Tyne, 2012).

\subsection{Le transcodage en laboratoire de langue}

L'activité de transcodage a été mise en application dans le cadre des Cours d'été de langue française de l'université de Genève ${ }^{20}$, en cours de prononciation en laboratoire, destiné à des apprenants de niveaux $\mathrm{B}$ et $\mathrm{C}$ du CECRL (Conseil de l'Europe, 2005) ${ }^{21} .87$ apprenants ${ }^{22}$ ont participé aux trois séances de transcodage, 12 hommes et 75 femmes, âgés en moyenne de 33 ans, de 27 pays différents, surtout d'Europe (65\%), mais aussi d'Amérique (27\%), d'Asie (7\%) et d'Afrique (2\%). Les L1 les plus représentées sont l'espagnol (24\%), suivi du suisse-allemand $(13 \%)$, de l'anglais $(11,5 \%)$, de l'italien (10\%), du russe $(9 \%)$ et du portugais $(6 \%)$. Pour le niveau de compétence, validé lors du test d'entrée, les apprenants se répartissent en trois groupes : niveau B1 (31\%), B2 $(30 \%), C(27 \%)$ et non renseigné $(11 \%)^{23}$.

20. Fondés en 1891, les Cours d'été attirent des apprenants du monde entier et offrent donc un terrain privilégié d'observation et d'enquête.

21. Nous avons renoncé pour le moment (sans l'exclure pour l'avenir) à tester cette activité avec des débutants (A1-A2). Nous estimons que le transcodage se prête à un éveil à la variation stylistique qui peut intervenir très tôt, même chez des débutants (voir Guimbretière, 2001; Dewaele \& Mougeon, 2002; Tyne, 2009b). Notre expérience didactique nous a montré que les apprenants perçoivent plus qu'on ne le croit.

22. Le nombre de 87 a été calculé compte non tenu de ceux qui ont participé à plusieurs séances, dont le nombre est difficile à établir. À cela s'ajoute l'anonymat des questionnaires d'évaluation et des fiches pédagogiques, censé favoriser la sincérité des réponses par l'absence d'enjeux évaluatifs.

23. Pour le niveau C, on n'a pas fait de distinction en $\mathrm{C} 1-\mathrm{C} 2$, parce que les Cours d'été ne la font pas. 


\subsection{Description du matériel et des supports didactiques}

Les trois extraits proposés, longs d'environ 30 secondes chacun, sont des interactions de jeunes locuteurs de la région parisienne (6 adolescents, 2 filles et 4 garçons; 2 locuteurs par extrait $)^{24}$, qui donnent à voir un français ordinaire d'échanges entre amis ou gens qui se connaissent bien, autour de thématiques de la vie courante (école, famille, loisirs, etc.) $)^{25}$.

Une fiche pédagogique papier, servant de base pour la transcription, est distribuée pour chaque séance, avec un titre et des illustrations contextualisant l'extrait sonore. Le schéma de l'interaction y est reproduit. Les tours de parole ont donc été rendus explicites pour simplifier une tâche couteuse en termes cognitifs. Un petit questionnaire anonyme est aussi distribué, pour recueillir les données socio-démographiques et obtenir l'avis des participants sur la difficulté et l'utilité de la tâche. Les apprenants devaient indiquer les raisons de la difficulté perçue, parmi : l'accent, le rythme, l'articulation, la mauvaise qualité de l'enregistrement, les chevauchements, le vocabulaire, le niveau de langue, la durée. Ils pouvaient ajouter des rubriques.

Enfin, deux questions ouvertes complètent le questionnaire, portant sur l'utilité de cette activité et sur ce qu'ils pensent avoir appris par le transcodage. Il s'agit à la fois de recueillir des remarques sur l'activité dans une optique qualitative et d'évaluer les retombées pédagogiques éventuelles.

\subsection{Déroulement global de l'activité}

Trois séances d'une trentaine de minutes se déroulent dans un laboratoire de langue équipé de 24 postes informatiques. Elles comportent cinq étapes : écoute globale, écoute détaillée, transcodage, confrontation et prise de conscience, réflexion-débat.

Les apprenants sont invités à écouter (individuellement) l'extrait sonore et à émettre des hypothèses. À partir de la «musique de la langue»,

24. Nous renvoyons, entre autres, à Guerin \& Paternostro (2014), pour une démythisation de la «langue des jeunes». Bien entendu, d'autres corpus de français parlé peuvent avoir toute leur place dans cette démarche d'initiation à la variation phonétique (voir conclusion).

25. Les traits phonétiques présents dans les extraits sont du même ordre que les phénomènes décrits en 2 . 
ils cherchent à imaginer le contexte de l'échange, l'identité des locuteurs, les liens qui les unissent.

Ensuite, ils sont appelés à réécouter l'extrait en portant leur attention sur les détails phoniques. Cette étape demande une écoute déjà plus attentive, même si c'est seulement au cours de la troisième étape, celle du transcodage proprement dit, qu'ils auront à se focaliser sur la compréhension du détail en passant à l'écrit ${ }^{26}$. Cette étape est suivie de la confrontation de la transcription de l'apprenant au corrigé fourni par l'enseignant.

Enfin, les apprenants peuvent réécouter le dialogue à loisir pour revenir sur ce qui a été observé ou pour vérifier ce qui n'a pas été compris. Cette étape est aussi l'occasion d'une réflexion-débat animée par l'enseignant ${ }^{27}$, autour des éléments qui ont surpris les apprenants.

L'activité s'achève par le questionnaire d'évaluation, individuel et anonyme.

\section{Analyses et discussion des résultats}

Nous ne nous attarderons pas sur ce que nous avons observé lors des séances de transcodage ni sur l'analyse des transcriptions. En général, les apprenants semblent avoir été surpris de découvrir que les natifs aussi hésitent, se répètent, amorcent des mots, se trompent. La découverte de la «fragilité» du natif semble avoir un effet bénéfique sur leur (in)sécurité linguistique.

Quant aux difficultés ressenties (fig. 1), le transcodage a été évalué comme difficile (54\%) mais pas impossible : $29 \%$ des apprenants l'ont jugé difficile à un degré (+), $25 \%$ à deux degrés $(++)$.

26. La transcription a été faite sur la fiche pédagogique papier décrite en 3.3.

27. L'enseignant et le chercheur sont la même personne. Cette activité a été proposée la dernière semaine de cours, vers la fin du parcours d'apprentissage. 


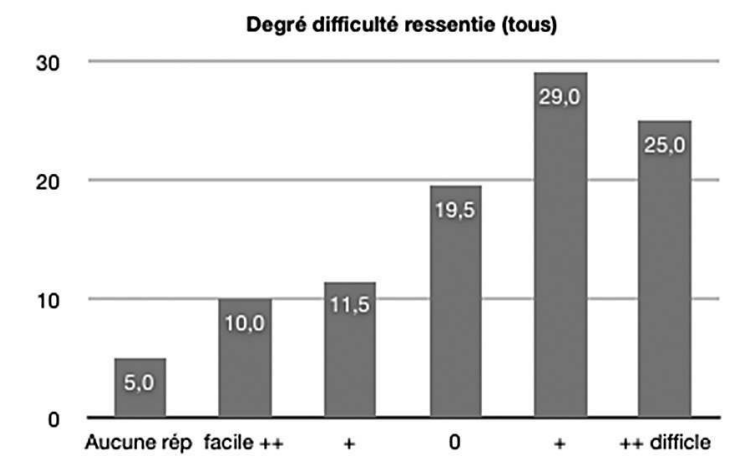

Fig. 1. - Tableau affichant le degré de difficulté ressentie.

Pour les difficultés caractérisant les trois extraits, les résultats (fig. 2) montrent que les éléments qui ont posé le plus de problèmes sont, par ordre décroissant : l'articulation, le rythme, l'accent, le registre familier, le vocabulaire, les tours de parole rapides. En revanche, ni la qualité sonore des extraits ni leur durée ne semblent avoir posé problème.

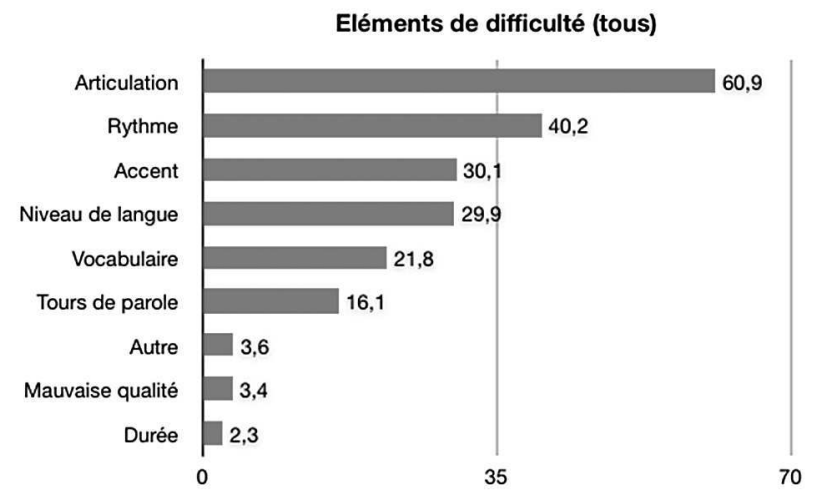

Fig. 2. - Pourcentage des éléments de difficulté présents dans les extraits.

Les étiquettes adoptées, parfois impressionnistes, ont été choisies par souci de clarté. L'articulation, par exemple, renvoie à ce que le sens commun entend en demandant de prononcer de façon claire et distincte. Ce qui, d'un point de vue acoustique, est plutôt lié au débit qu'aux gestes articulatoires. De même, le terme «accent» est utilisé au sens courant 
de façon de parler sur laquelle les locuteurs portent un jugement social, etc.

Quant à l'utilité du transcodage pour un éveil à la variation phonétique, $93 \%$ des apprenants le considèrent comme utile. La difficulté de la tâche ne les a donc pas empêchés d'en voir l'utilité.

En ce qui concerne les questions ouvertes, la première porte sur l'utilité de l'intégralité de l'activité sans se focaliser sur le transcodage. Une approche qualitative nous permet de regrouper les réponses en 4 catégories : (1) Prise de conscience et reconnaissance; (2) Entrainement et effort; (3) Nouveauté et pertinence; (4) Difficulté.

Pour (1), la quasi-totalité des réponses identifie le transcodage à une prise de conscience :

(1.1) Quand je dois écrire je comprends mieux. Et tous les jours on doit comprendre pas seulement qui parle le français très lentement mais aussi qui parle vite et avec un niveau de langue très familier ${ }^{28}$.

(1.2) Utile, voir différent prononciation des mots 'standard'.

(1.3) On peut nous rendre compte de comment les français parlent dans certains occasions pour reconnaitre le différent accents et aussi pour savoir l'interaction et vélocité.

Les apprenants estiment utile de se familiariser avec la façon dont les francophones parlent en situation ordinaire. Ils se rendent compte, en effet, qu'il y a des locuteurs qui parlent rapidement et qui usent d'une langue familière. Le transcodage leur permet de «voir» les différentes façons dont les mots du standard sont prononcés. L'emploi du verbe «voir» (1.2) nous parait significatif. En tant qu'observatoire privilégié sur la variation phonétique, le transcodage exploite le passage d'une réalité sonore (l'oral) à une réalité visuelle (l'écrit), pour aider les apprenants à reconnaitre les différents accents et à décrypter les interactions rapides.

Pour (2), l'idée d'effort est associée à l'écoute dans la quasi-totalité des réponses :

(2.1) C'est la forme d'améliorer l'écoute. C'est vraiment pédagogique.

(2.2) M'entraîner à comprendre la langue parlée normalement et dans la rue, d'une façon rapide.

28. Seules les réponses les plus significatives sont reportées ici, reproduites telles que rédigées. 
(2.3) Prêter un plus d'attention aux finesses de la langue.

(2.4) On s'habitue l'oreille aux accents différents.

(2.5) Mon compréhension oral a s'élevé un peu.

L'effort est donc associé à l'écoute et non au transcodage, comme la difficulté perçue aurait pu le suggérer. On s'entraine pour améliorer l'écoute, le but étant de comprendre la langue ordinaire parlée dans la rue, de familiariser l'oreille aux accents divers et aux subtilités de la langue.

Pour (3), les apprenants affirment avoir appris quelque chose de nouveau et de pertinent, notamment pour les interactions de la vie quotidienne :

(3.1) J'ai écouté quelque chose différent qui existe dans mon vie quotidienne.

(3.2) J'ai souvent des problèmes à comprendre les gens francophones qui ne sont pas professeurs. Les profs parlent plus clairement.

(3.3) Oui, c'était utile parce que j'ai appris le langage qu'on attend dans la rue ou en conversation. Maintenant je peux comprendre un peu mieux qu'est-ce qu'ils discutent dans le tram.

(3.4) On apprend les mots comment ils sont parlés (ou comme on les écoute) p. ex. je suis $->$ chuis.

(3.5) Elle présent la langue parlée par des gens ordinaires. Ça aide à comprendre certaine prononciation de certains mots quand ils sont raccourcis.

Ils semblent avoir saisi le cœur de l'activité : le transcodage leur a permis de se familiariser avec l'accent de situations informelles de la vie ordinaire. Ils disent mieux comprendre, par exemple, les gens qui conversent dans le tram ou dans la rue. Le transcodage s'est aussi avéré pertinent par rapport à leurs besoins. L'un d'entre eux affirme que les extraits lui permettent de mieux comprendre les locuteurs qui ne parlent pas comme des professeurs, d'où le besoin d'entrer en contact avec une langue de locuteurs ordinaires, proche de la vie réelle, moins artificielle que celle actualisée en classe.

Pour (4), les apprenants ont mis en évidence quelques difficultés rencontrées lors du transcodage :

(4.1) Il y avait des phrases que je n'ai pas bien compris parce qu'ils était combiné en un mot.

(4.2) C'était long. 
(4.3) C'était dur à suivre. Mon français est très 'académique et standard' donc pour moi a été quelque chose de nouveau. Intéressant mais difficile.

(4.4) Niveau trop haut.

Les principales difficultés sont la durée, la difficulté à comprendre les consignes, et un niveau de langue trop complexe. Le français académique et standard semble constituer un obstacle (4.3). Ils n'ont que rarement l'occasion d'entendre un français courant, face auquel ils sont déroutés. C'est ce constat qui fait que malgré la difficulté, l'activité de transcodage a été perçue comme utile et intéressante. D'une certaine façon, les apprenants ont compris que la difficulté va de pair avec l'intérêt et l'efficacité.

Contrairement à la première question, la deuxième se focalise sur l'étape du transcodage.

(5.1) Ça m'a permis de mieux comprendre aussi l'oral que à l'écriture. Mettre les sons en parole, ça donne une structure intégral qui permet de faire aller-retour. À fur et à mesure que on comprend, on reconnaît mieux les sons, l'intonation, les liaisons, l'accent, l'intention, le ton amical, formel. On se fait une image du contexte dans lequel se déroule la conversation.

(5.2) Oui la vie quotidienne aux banlieue, la vision des gens qui habitent là-bas. Comprendre des sentiments, intonations et surtout le message. Toujours intéressant.

(5.3) La transcription m'a fait concentré sur les mots et les phrases.

(5.4) Il est vraiment possible d'entendre et comprendre presque tous les mots en les écoutant plusieurs fois! Cela est très satisfaisant! :-).

En essayant de résumer les commentaires recueillis, nous pouvons souligner que le fait de «mettre les sons en paroles» (5.1) aide les apprenants à se focaliser «sur les mots et les phrases» (5.3), c'est-à-dire sur les phones et les suites de phones. Au fur et à mesure qu'ils comprennent, ils se rendent compte de traits de prononciation spécifiques, de particularités intonatives et de la continuité de la chaine parlée. À partir de ces détails, ils posent des hypothèses et mettent en place des stratégies d'interprétation de la situation, du ton de la conversation, etc. Les différents traits phonétiques dans une situation relevant de la proximité sont ainsi observés en situation.

À travers le transcodage, les apprenants prennent conscience des spécificités de la langue parlée. Ils réalisent que le français est une 
langue vivante, comme leur langue maternelle (même si un travail spécifique sur le lien entre le français et leur langue première n'a pas pu être fait, vu le nombre important de L1 présentes). L'observation en contexte leur permet aussi d'accéder à la dimension culturelle de l'échange.

\section{Conclusion}

Contrairement à ce qui se passe pour d'autres langues européennes, où la variation diastratique et/ou diatopique semble l'emporter sur le diaphasique ${ }^{29}$, c'est la variation stylistique qui prime en français (Gadet, 2007). Bien que cette affirmation mériterait sans doute d'être nuancée dans le contexte complexe de l'apprentissage du FLE, ce primat du diaphasique se heurte au manque de description des traits mobilisés ainsi qu'à la rareté des corpus oraux sensibles à la variation stylistique, collectés compte tenu de l'écologie des évènements discursifs.

À cela s'ajoute l'absence de réflexion sur le rôle du diaphasique dans l'enseignement du français. "L'évaluation diaphasique peut prendre la forme de nuances fines, alors même que les éléments linguistiques en jeu sont limités. (Gadet, 2001, p. 66) Ces nuances deviennent presque invisibles quand la variation phonétique entre en ligne de compte, c'est pourquoi le plan phonique est laissé à l'écart. Ce que le locuteur de français L1 possède par imprégnation, le locuteur de français L2 doit le construire par l'observation et l'apprentissage.

Fonctionnant comme une loupe et un catalyseur perceptif, le transcodage a permis la mise en place de passerelles hypothético-déductives entre oral et écrit, aidant les apprenants à se focaliser sur la prononciation ordinaire en contexte et favorisant la constitution d'une compétence pragmatique (Dewaele \& Mougeon, 2002).

Le choix d'extraits authentiques du corpus MPF n'a pas posé de problème particulier. Au contraire, ils semblent avoir retenu l'attention des apprenants, qui en ont apprécié l'utilité pour les échanges quotidiens en français. L'intégration d'autres corpus de français parlé pourrait permettre d'élargir la palette des actualisations possibles de la langue, témoignant d'autres situations de communication, en France et en francophonie.

29. Voir, entre autres, Armstrong (2002) pour la Grande-Bretagne, où la variation socio-régionale l'emporte sur le diaphasique, et Berruto (2005) pour l'Italie, où la diatopie est au cœur de la variation. 
Malgré le caractère exploratoire de cette application didactique, les résultats observés ainsi que les réponses obtenues nous amènent à dresser un bilan plutôt positif de cette expérience. Néanmoins, la réflexion mérite d'être poursuivie et approfondie, notamment en ce qui concerne la prise en compte, d'une part, des problématiques psycholinguistiques liées à l'apprentissage du système orthographique du français et à la question délicate du décalage entre la graphie et la phonie. D'autre part, il serait intéressant d'observer, sur le long terme, ce que les apprenants font de leur «découverte» de la langue ordinaire en dehors du contexte d'apprentissage, particulièrement en production.

\section{RÉFÉRENCES BIBLIOGRAPHIQUES}

AndRÉ, Virginie \& Tyne, Henry. (2012). Compétence sociolinguistique et dysfluence en L2. Dans A. Kamber \& C. Skupien (dir.), Recherches récentes en FLE. Bern : Peter Lang.

ArmStrong, Nigel. (2002). Nivellement et standardisation en anglais et en français. Langage et Société, 102, 5-32.

Lodge, Robert Anthony, Armstrong, Nigel, Ellis, Yvette \& Shelton, Jane. (1997). Exploring the French Language. Londres : Arnold.

Berruto, Gaetano. (2003). Sul parlante nativo (di italiano). Dans H. Radatz \& S. Schlösser (dir.). Donum Grammaticorum. Festschrift für Harro Stammerjohann (p. 1-14). Tübingen : Niemeyer.

Berruto, Gaetano. (2005). Fondamenti di sociolinguistica. Bari : Laterza.

BIGGS, Patricia \& Dalwood, Mary. (1976). Les Orléanais ont la parole. Teaching Guide and Tapescript. Londres : Longman.

Boulton, Alex \& Tyne, Henry. (2014). Des documents authentiques aux corpus. Démarches pour l'apprentissage des langues. Paris : Didier.

Callamand, Monique. (1973). L'intonation expressive. Paris : Hachette.

Charliac, Lucille \& Motron, Annie-Claude. (2006). Phonétique progressive du français. Niveau avancé. Paris : CLE international.

CONSEIL DE L'EUROPE. (2005). Cadre européen commun de référence pour les langues. Paris : Didier.

Dewaele, Jean-Marc \& Mougeon, Raymond (dir.). (2002). L'appropriation de la variation sociolinguistique par les apprenants avancés du FL2 et FLE. Acquisition et Interaction en Langue Étrangère, 17.

Debaisieux, Jeanne-Marie \& Boulton, Alex. (2007). Alors la question c'est...? Questions pragmatiques et annotation pédagogique des corpus. Cahiers AFLS, 13(2), 31-59. Disponible en ligne sur <www.afls.net/ cahiers/cover13.2.php> (consulté le 12 décembre 2013). 
Detey, Sylvain. (2010). Normes pédagogiques et corpus oraux en FLE : le curseur apprenabilité/acceptabilité et la variation phonético-phonologique dans l'espace francophone. Dans B. Olivier \& I. Schaffner (dir.), Quel français enseigner? La question de la norme dans l'enseignement/ apprentissage (p. 155-168). Palaiseau : Éditions de l'École polytechnique.

Detey, Sylvain, Durand, Jacques, Laks, Bernard \& Lyche, Chantal. (2010). Les variétés du français parlé dans l'espace francophone. Ressources pour l'enseignement. Paris : Ophrys.

Detey, Sylvain \& Racine, Isabelle. (2012). Les apprenants de français face aux normes de prononciation : quelle(s) entrée(s) pour quelle(s) sortie(s)? Revue française de linguistique appliquée, 17(1), 81-96.

GAdet, Françoise. (1997). Le français ordinaire (2 éd.). Paris : Armand Colin.

GADET, Françoise. (2001). Enseigner le style. Le français dans le monde. Recherches et applications (numéro spécial), 63-68.

GADET, Françoise. (2007). La variation sociale en français (2e éd.). Paris : Ophrys.

Gadet, Françoise. (2012). Les locuteurs et les savoirs sur les langues. Le français aujourd'hui, 176, 123-126.

Gadet, Françoise \& Guerin, Emmanuelle. (2012). Des données pour étudier la variation : petits gestes méthodologiques, gros effets. Cahiers de linguistique, 38(1), 41-65.

Gadet, Françoise \& Paternostro, Roberto. (2013). Un accent multiculturel en région parisienne? Repères DoRiF. Disponible en ligne sur $<w w w . d o r i f . i t / e z i n e / e z i n e \_a r t i c l e s . p h p$ ?dorif_ezine=a4211572e6fb6bb c0755b986fe03ab91\&art_id=94> (consulté le 12 décembre 2013).

Galazzi, Enrica. (2002). Le son à l'école. Phonétique et enseignement des langues (fin XIX siècle-début $X X^{e}$ siècle). Brescia : La Scuola.

GuERIN, Emmanuelle. (2008). 'Le français standard' : une variété située? Actes du Congrès mondial de linguistique française, 8, 2303-2312.

GuERIN, Emmanuelle. (2010). L'«outre-langue» des enseignants ou le mythe d'une langue monovariétale. Pratiques, 145-146, 45-54.

Guerin, Emmanuelle \& Paternostro, Roberto. (2014). What Is langue des jeunes and Who Speaks It? Dans H. Tyne et coll. (dir.), Ecological and Data-Driven Perspectives in French Language Studies. Newcastle : Cambridge Scholars Publishing.

Guimbretière, Élisabeth. (1992). Paroles : entraînement à la compréhension et à l'expression orales. Paris : Didier.

Johns, Tim. (1993). Data-Driven Learning: An Update. TELL\&CALL, 2, 4-10. 
Koch, Peter \& Esterreicher, Wulf. (2001). Langage parlé et langage écrit. Dans G. Holtus, M. Metzeltin \& C. Schmitt (dir.), Lexikon der Romanistischen Linguistik (tome 1, p. 584-627). Tübingen : Max Niemeyer Verlag.

Lebre-Peytard, Monique, Lieutaud, Simone, Beacco, Jean-Claude \& Malandain, Jean-Louis. (1977). Le document oral brut dans la classe de français : la transcription de documents sonores authentiques. Paris : BELC.

LÉON, Pierre. (1993). Précis de phonostylistique. Parole et expressivité. Paris : Nathan.

Lноте, Élisabeth (dir.). (1990). Le paysage sonore d'une langue : le français. Hambourg : Buske Verlag.

Lноте, Élisabeth. (1995). Enseignement de l'oral en interaction. Paris : Hachette FLE.

LyNCH, Tony. (2007). Learning from the Transcripts of an Oral Communication Task. ELT Journal, 61(4), 311-320.

Paternostro, Roberto. (2014). L'intonation des jeunes en région parisienne : aspects phonétiques et sociolinguistiques, implications didactiques (Thèse de doctorat : Università di Brescia, Italie \& Université Paris Ouest, France).

Pimsleur, Paul. (1979). Le pont sonore. Paris : Hachette.

Regan, Vera, Lemee, Isabelle \& Howard, Martin (2009). The Acquisition of Sociolinguistic Competence in a Study Abroad Context. Londres : Multilingual Matters.

Selting, Margaret. (1994). Emphatic Speech Style: With Special Focus on the Prosodic Signalling of Heightened Emotive Involvement in Conversation. Journal of Pragmatics, 22, 375-408.

Stillwell, Christopher et coll. (2010). Students Transcribing Tasks: Noticing Fluency, Accuracy, and Complexity. ELT Journal, 64(4), 445-455.

Tyne, Henry. (2009a). Corpus oraux par et pour l'apprenant. Mélanges CRAPEL, 31, 91-111. Disponible en ligne sur <www.atilf.fr/IMG/pdf/ melanges/05_Tyne.pdf>.

Tyne, Henry. (2009b). Style in L2: The Icing on the Cake? Dans E. Labeau \& F. Myles. (dir.), The Advanced Learner Variety: The Case of French (p. 243-268). Bern : Peter Lang.

Tyne, Henry. (2012). Corpus Work with Ordinary Teachers: Data-Driven Learning Activities. Dans A. Boulton \& J. Thomas (dir.), Teaching and Language Corpora: Input, Process and Product. Selected papers from TaLC9. Brno, République tchèque : Masaryk University Press.

Valdman, Albert. (1989). The Elaboration of Pedagogical Norms for Second Language Learners in a Conflictual Diglossia Situation. Dans S. Gass, C. Madden, D. Preston \& L. Selinker (dir.), Variation in 
Second Language Acquisition (vol. 1, p. 15-34). Clevedon, RoyaumeUni : Multilingual Matters.

Valdman, Albert. (2000). Comment gérer la variation dans l'enseignement du français langue étrangère aux États-Unis. The French Review, 73(4), 648-66. 